\title{
On the analysis of a dynamical model representing gene regulatory networks under negative feedback
}

\author{
M. E. Ahsen ${ }^{1}$, H. Özbay ${ }^{2, * \dagger}$ and S-I. Niculescu ${ }^{3}$ \\ ${ }^{1}$ Department of Bioengineering, University of Texas at Dallas, Richardson, TX 75080-3021, USA \\ ${ }^{2}$ Department of Electrical and Electronics Engineering, Bilkent University, Ankara TR-06800, Turkey \\ ${ }^{3}$ Laboratoire des Signaux et Systèmes (UMR CNRS 8506) CNRS-SUPELEC, 91192, Gif-sur-Yvette, France
}

\begin{abstract}
SUMMARY
In this work, stability analysis is performed for a cyclic dynamical model of gene regulatory networks involving time delays, under negative feedback. The model considered has nonlinearities with negative Schwarzian derivatives. Sufficient conditions implying global stability of these types of GRNs are obtained. The special case of homogenous gene regulatory networks is also studied; in this case, the proposed stability conditions depend only on the parameters of the nonlinearity function. Illustrative numerical examples complete the presentation. Copyright () 2013 John Wiley \& Sons, Ltd.
\end{abstract}

Received 13 February 2012; Revised 1 October 2012; Accepted 16 November 2012

KEY WORDS: gene regulatory networks; Schwarzian derivatives; asymptotic stability; hill functions; time delay

\section{INTRODUCTION}

Recent advances in micro-array technology have facilitated measurement of expression levels of genes [1]. As a consequence, with the abundance of gene expression data, our understanding of the underlying mechanism in gene regulation has increased enormously. Basically, a gene regulatory network (abbreviated as GRN in the sequel) can be described as the interaction of DNA segments with themselves and with regulatory proteins in the cell. Therefore, it can be thought as an indicator of the transcription rates of the genes into mRNA, which is used for delivering the coding information required for the protein synthesis [2]. Gene regulation has been extensively studied in the literature in the last two decades as it has been thought that malfunction in the regulation of genes may lead to serious diseases including cancer [3]. There are two basic models to study gene regulation: (i) boolean model and (ii) dynamical model (see [4] and the references therein for a literature review on both models).

This paper investigates global stability of a cyclic dynamical model accounting for the GRN including a nonlinear feedback loop and time delays. The dynamical model studied is described in Figure 1.

Here $G_{i}$ is a stable first-order filter whose input is a nonlinear function of the delayed output of $P_{i}$. Similarly, $P_{i}$ is a stable first-order system whose input is a nonlinear function of the delayed output of $G_{i-1}$ for $1 \leqslant i<m-1$, and $P_{1}$ has an input that is a nonlinear function of the delayed output of $G_{m}$. The model proposed in [5] consists of a set of differential equations in the

\footnotetext{
*Correspondence to: Hitay Özbay, Department of Electrical and Electronics Engineering, Bilkent University, Ankara TR-06800, Turkey.

†E-mail: hitay@bilkent.edu.tr 


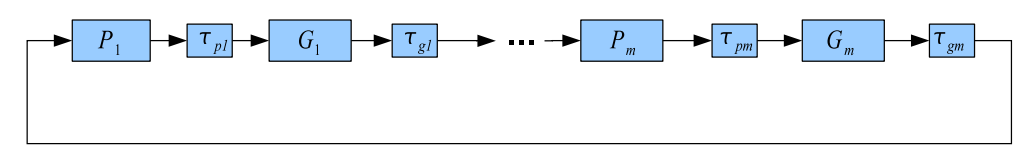

Figure 1. A continuous time model of gene regulatory network.

following form:

$$
\left\{\begin{aligned}
\dot{p}_{1}(t) & =-k_{p 1} p_{1}(t)+f_{p 1}\left(g_{m}\left(t-\tau_{g m}\right)\right) \\
\dot{g}_{1}(t) & =-k_{g 1} g_{1}(t)+f_{g 1}\left(p_{1}\left(t-\tau_{p 1}\right)\right) \\
& \vdots \\
\dot{p}_{m}(t) & =-k_{p m} p_{m}(t)+f_{p m}\left(g_{m-1}\left(t-\tau_{g_{m-1}}\right)\right) \\
\dot{g}_{m}(t) & =-k_{g 1} g_{m}(t)+f_{g m}\left(p_{m}\left(t-\tau_{p m}\right)\right)
\end{aligned}\right.
$$

with appropriate initial conditions, where $p_{i}$ and $g_{i}$ represent the protein and mRNA concentrations, respectively. Models in this general structure are frequently encountered in the modeling of biological processes such as mitogen-activated protein cascades and circadian rhythm generator; see, for example, [6-8] and [9]. To account for the switch-like phenomena observed in gene regulation, the nonlinear regulation functions are often approximated by Hill functions [4, 10]. In [5], system (1) is analyzed, and a local stability result is given by including explicit information on the value of the time delay. Again, for the local stability of this system, an explicit computation of the upper bound of the delay value is performed in [11]. For GRNs, a mathematical modeling work has been done in [12], where Hill functions are taken as nonlinearities, and the coefficients of specific Hill functions are estimated from experimental data. It is worth mentioning that mathematical models similar to (1) are also found in the neural networks literature. For example, in [8], system (1) has been considered with nonlinearities as tangent hyperbolic functions.

In this work, we will assume that the functions $f_{i}$ are nonlinear and have negative Schwarzian derivatives. Functions with negative Schwarzian derivatives include Hill functions and tangent hyperbolic functions. A linear model of repressilator (a special type of GRN) has been analyzed in [13] by using the Schwarzian derivative concept. System (1) under single time delay and negative feedback has been studied in [14], where an easy condition for guaranteeing asymptotic stability has been obtained by using the arguments of $[15,16]$ to embed the original system (1) to a discretetime system. By using a Hopf bifurcation approach, Enciso [14] shows existence of oscillations for some cases.

In this paper, we perform a global stability analysis of the GRN. In particular, a necessary and sufficient condition is derived for the delay-independent global stability of (1). Moreover, by using a Poincaré-Bendixson type result, we show in this paper that when the global stability condition is not satisfied, instability is in the form of periodic oscillations. Furthermore, upper and lower bounds on the magnitude of the periodic oscillations are estimated for the corresponding unstable GRNs. All of these results are obtained under the assumption that the nonlinear functions have negative Schwarzian derivatives. When all the nonlinearities in (1) are the same, we say that the system is a homogenous GRN. For some typical Hill function choices for these nonlinearities, global stability condition is expressed in terms of the parameters of these functions.

The rest of the paper is organized as follows. In Section 2, notations as well as the problem formulation are given. In Section 3, we introduce some preliminary results to be used in the stability analysis. In Section 4, we exploit the negative Schwarzian derivative property of functions and prove that such functions have two specific forms. On the basis of this classification result, we present results regarding the number of fixed points of such functions. In Section 5, the main results are presented. As a special case of the negative feedback, in Section 6 the homogenous GRN is considered. Examples are given in Section 7, and concluding remarks end the paper. 


\section{NOTATION, PRELIMINARIES AND PROBLEM FORMULATION}

In this section, we present some basic definitions and notations that are frequently used in this work.

The notation $f^{m}$ stands for the function obtained by $m$ compositions of a given function $f$. We say that $x$ is a fixed point of $f$ if it satisfies $f(x)=x$. The symbol $D^{n}\left(\mathbb{R}_{+}\right)$denotes the set of $n$ times continuously differentiable functions.

Let $z(t)$ be a vector function depending on the variable $t>0$. A point $w \in \mathbb{R}^{n}$ is said to be an omega point of $z(t)$ if there is an increasing sequence $t_{i} \rightarrow \infty$ such that

$$
\lim _{t_{i} \rightarrow \infty}\left(z\left(t_{i}\right)\right)=w .
$$

The omega limit set of $z(t)$ is the set of all its omega points.

For the analysis of (1), we will use properties of Schwarzian derivatives, which are commonly employed in analysis of these types of cyclic nonlinear feedback systems; see, for example, [13]. Let a function $f$ be defined from $\mathbb{R}_{+}$to $\mathbb{R}_{+}$. Suppose $f$ is at least three times continuously differentiable, with $f^{\prime}, f^{\prime \prime}$ and $f^{\prime \prime \prime}$ representing its first, second and third derivatives, respectively. Then, the Schwarzian derivative of the function $f$ - see [17], denoted as $S f(x)$ - is given by the following:

$$
S f(x)= \begin{cases}-\infty & \text { if } f^{\prime}(x)=0 \\ \frac{f^{\prime \prime \prime}(x)}{f^{\prime}(x)}-\frac{3}{2}\left(\frac{f^{\prime \prime}(x)}{f^{\prime}(x)}\right)^{2} & \text { if } f^{\prime}(x) \neq 0\end{cases}
$$

In this paper, we analyze the following simplified system, which is equivalent to (1), where we have a single delay, $\tau>0$, in the feedback channel:

$$
\left\{\begin{aligned}
\dot{x}_{1}(t) & =-\lambda_{1} x_{1}(t)+g_{1}\left(x_{2}(t)\right) \\
\dot{x}_{2}(t) & =-\lambda_{2} x_{2}(t)+g_{2}\left(x_{3}(t)\right) \\
& \vdots \\
\dot{x}_{n}(t) & =-\lambda_{n} x_{n}(t)+g_{n}\left(x_{1}(t-\tau)\right) .
\end{aligned}\right.
$$

In the sequel, we present conditions for the asymptotic stability and existence of oscillations regarding the nonlinear time delayed feedback system (3) under the following simplifying assumptions:

\section{Assumption 1}

For all $i=1,2, \ldots, n$, we have $\lambda_{i}>0$.

Assumption 2

For all $i=1,2, \ldots, n$, the nonlinearity functions $g_{i}$ satisfy the following:

(i) $g_{i}(x)$ is a bounded function defined on $\mathbb{R}_{+}$;

(ii) we have either

$$
g_{i}^{\prime}(x)<0 \quad \text { or } \quad g_{i}^{\prime}(x)>0 \quad \forall x \in(0, \infty) .
$$

(iii) Each function $g_{i}$ has $S g_{i}(x)<0 \quad \forall x \in(0, \infty)$.

Assumption 2 simply means that each $g_{i}$ is a monotone function and takes positive values. The nonlinearity functions have $\mathbb{R}_{+}$as their domain because their domain represents biological variables, which take positive values. In Section 1, we have said that the nonlinearity functions in the proposed model are either Hill functions or tangent hyperbolic function; hence, they are bounded and monotonic. Therefore, these assumptions do not impose any constraints on our analysis of the GRN, and they are compatible with various modeling studies; see, e.g., [12]. Also note that $g_{i}^{\prime}(0)=0$ 
is allowed, because it does not violate the monotonicity of $g_{i}$. We will now define a new function $g$ in the following way:

$$
g=\left(\frac{1}{\lambda_{1}} g_{1}\right) \circ\left(\frac{1}{\lambda_{2}} g_{2}\right) \circ \cdots \circ\left(\frac{1}{\lambda_{n}} g_{n}\right) .
$$

We say that the GRN is under negative feedback if

$$
g^{\prime}(x)<0 \quad \forall x \in(0, \infty) .
$$

Conversely, the GRN is said to be under positive feedback if the this inequality is reversed. In this work, we will only be concerned with the negative feedback case.

Later, we will make use of the following Poincaré-Bendixson type theorem from [18]:

\section{Theorem 1}

Suppose that the system (3) is under negative feedback with its unique equilibrium point $x_{e q}=$ $\left(x_{1}, \ldots, x_{n}\right)$. Let $x(t)$ be a solution of the system (3), which is bounded in $\mathbb{R}_{+}^{n}$. Then, the omega limit set of $x(t)$ consists of either

(i) an equilibrium point or

(ii) a non-constant periodic orbit.

In section 5, we will show that under Assumptions 1 and 2, every solution of the system remains bounded in $\mathbb{R}_{+}^{n}$. Therefore, the solutions of the system (3) either converge to the unique equilibrium point or are periodic.

\section{SOME PRELIMINARY RESULTS}

Recall the definition of the Schwarzian derivative of a function $f$ given in (2). In this paper, we are dealing with functions satisfying one of the following conditions:

$$
f^{\prime}(x)>0 \quad \text { or } \quad f^{\prime}(x)<0 \quad \forall x \in(0, \infty) .
$$

Therefore, $S f(x)>-\infty$ for the class of functions we are interested in. Some immediate results can be deduced from the definition (2) as follows:

\section{Lemma 1}

Let $I \subseteq R$ be an interval and suppose $f, g \in D^{3}\left(\mathbb{R}_{+}\right)$such that the function $f \circ g(x)$ is well defined. Suppose also that we have

$$
f^{\prime}(x) \neq 0 \quad \forall x \in(0, \infty)
$$

then, the following properties hold:

1. For any $c \in \mathbb{R}$ and $d \in \mathbb{R} \backslash\{0\}, S f(x)=S(f(x)+c)$ and $S f(x)=S(d f(x))$.

2. $S(f \circ g)(x)=S f(g(x)) \cdot g^{\prime}(x)^{2}+S g(x)$.

3. If $S f(x) \leqslant 0, S g(x)<0$, then $S(f \circ g)(x)<0$.

4. If $S f(x)<0 \forall x \in \operatorname{int}(I)$, then $f^{\prime}(x)$ cannot have positive local minima nor negative local maxima.

The proofs of the properties mentioned in Lemma 1 can be found in [17]. From (2), we can calculate Schwarzian derivatives of some functions that are frequently used in the analysis of biological systems as follows:

$$
\begin{gathered}
S\left(\frac{a}{b+x^{m}}\right)=S\left(\frac{a x^{m}}{b+x^{m}}\right)=-\frac{\left(m^{2}-1\right)}{x^{2}} \\
S(p \tanh (q x))=-2 q^{2} \quad a, b, p, q \geqslant 0 \quad m \in \mathbb{N} .
\end{gathered}
$$

We can see that Hill functions, (8), have negative Schwarzian derivatives for $m \geqslant 2$. 
It is a well-known fact that a decreasing, continuous function $f$ defined on $\mathbb{R}_{+}$taking positive values has exactly one fixed point, which leads to the following result whose detailed proof can be found in [19].

Lemma 2

Let $h(x): \mathbb{R}_{+}^{n} \rightarrow Y \subseteq \mathbb{R}_{+}^{n}$ be defined as

$$
h\left(x_{1}, x_{2}, \ldots, x_{n}\right)=\left(\begin{array}{c}
h_{1}\left(x_{2}\right) \\
\vdots \\
h_{n-1}\left(x_{n}\right) \\
h_{n}\left(x_{1}\right)
\end{array}\right)
$$

where

$$
h_{i}\left(z_{i}\right): \mathbb{R}_{+} \rightarrow Y_{i} \subseteq \mathbb{R}_{+} \quad \forall i=1,2, \ldots, n .
$$

Let the function $q(t)$ from $\mathbb{R}_{+}$to $Y_{1} \subseteq \mathbb{R}_{+}$be defined as

$$
q(t)=h_{1} \circ h_{2} \circ \ldots \circ h_{n}(t) .
$$

There is a bijection between the fixed points of $h$ and those of $q$. In particular, if $q$ is a decreasing function or if we have

$$
\left|h_{i}^{\prime}(z)\right|<1 \quad \forall z \in \mathbb{R}_{+} \quad \forall i=1, \ldots, m,
$$

then the function $h$ has a unique fixed point.

Proof

For the proof, we refer to [19], where the following observation is exploited: for each fixed point $x$ of the function $q$, the point $u=\left(x, h_{2} \circ \ldots \circ h_{n}(x), \ldots, h_{n}(x)\right)$ is a fixed point of the function $h$.

\section{PROPERTIES OF FUNCTIONS WITH NEGATIVE SCHWARZIAN DERIVATIVES}

In this section, we will prove a classification result for functions with negative Schwarzian derivatives. For that purpose, we need the following preliminary result:

\section{Lemma 3}

Let $h$ be a three times differentiable function from $\mathbb{R}_{+}$to $Y \subseteq \mathbb{R}_{+}$and suppose that we have

$$
-\infty<\operatorname{Sh}(x)<0, \quad \forall x \in(0, \infty) .
$$

Then, $h^{\prime}$ cannot be constant for any $[a, b] \subseteq(0, \infty)$ with $a<b$. Moreover, suppose that

$$
h^{\prime}(x)>0 \quad \forall x \in(0, \infty),
$$

and there exists $c \in \mathbb{R}_{+}$such that $h^{\prime \prime}(c)<0$; then we have

$$
h^{\prime \prime}(d) \leqslant 0, \quad \forall d \geqslant c .
$$

Proof

For the first part of Lemma, suppose on the contrary that there exists positive constants $a<b$ such that $h^{\prime}$ is constant in $[a, b]$. Letting $c \in(a, b)$, then $h^{\prime \prime}(c)=0=h^{\prime \prime \prime}(c)$; but this implies that

$$
\operatorname{Sh}(c)=0,
$$


which is a contradiction. Therefore, $h^{\prime}$ cannot be constant in any subinterval of $\mathbb{R}_{+}$. For the second part of Lemma, suppose that there exists positive real numbers $c<d$ such that $h^{\prime \prime}(c)<0$ and $h^{\prime \prime}(d)>0$. Let $I$ be defined as $I=[c, d]$. Because $h^{\prime}$ is a continuous function and $I$ is a compact set $\exists x_{1}, x_{2} \in I$ such that we have $h^{\prime}\left(x_{1}\right) \leqslant h^{\prime}(x) \leqslant h^{\prime}\left(x_{2}\right), \forall x \in I$.

But because $h^{\prime \prime}(c)<0, \exists y \geqslant c$ satisfying

$$
h^{\prime}(y)<h^{\prime}(c) .
$$

Similarly, because $h^{\prime \prime}(d)>0 \quad \exists z \leqslant d$ satisfying

$$
h^{\prime}(z)<h^{\prime}(d) .
$$

Equations (14) and (15) implies that $x_{1} \neq c, x_{1} \neq d$ and we have $h^{\prime}\left(x_{1}\right) \leqslant h^{\prime}(x) \forall x \in I$. Hence, by definition, $x_{1}$ is a positive local minima of the function $h^{\prime}$. But because $\operatorname{Sh}(x)<0, h^{\prime}$ cannot have a positive local minima. Therefore, $d \geqslant c$ implies $h^{\prime \prime}(d) \leqslant 0$.

Suppose that a function $h$, having the technical assumptions of Lemma 3, satisfies

$$
h^{\prime \prime}(y)=0, \quad h^{\prime}(y)>0
$$

for some $y \in(0, \infty)$. Then we have

$$
\begin{aligned}
S h(y) & =\frac{h^{\prime \prime \prime}(y)}{h^{\prime}(y)}-\frac{3}{2}\left(\frac{h^{\prime \prime}(y)}{h^{\prime}(y)}\right)^{2} \\
& =\frac{h^{\prime \prime \prime}(y)}{h^{\prime}(y)}<0 \\
& \Rightarrow h^{\prime \prime \prime}(y)<0,
\end{aligned}
$$

which implies that the point $y$ is a positive local maxima of the function $h^{\prime}$. Combining Lemmas 3 and (19), we can conclude that if $h^{\prime}$ will be decreasing in some interval $[a, b]$, then it will be decreasing in $[b, \infty]$. In particular, if $h^{\prime \prime}(0)<0$, then $h^{\prime \prime}(x) \leqslant 0$ for all $x \geqslant 0$, which implies that $h^{\prime}(x)$ is a decreasing function. Combining this fact with Lemma 3, we obtain the following result:

Corollary 1

Let $h$ be a three times differentiable function defined from $\mathbb{R}_{+}$to $Y \subseteq \mathbb{R}_{+}$and suppose that we have

$$
\operatorname{Sh}(x)<0 \quad \text { and } \quad h^{\prime}(x)>0, \quad \forall x \in(0, \infty)
$$

Then, $h^{\prime}$ is a function from $\mathbb{R}_{+}$to $Y \subseteq \mathbb{R}_{+}$satisfying one of the following properties:

1. $h^{\prime}$ is a strictly increasing function on $[0, \infty)$.

2. $h^{\prime}$ is a strictly decreasing function on $[0, \infty)$.

3. There exists $a \geqslant 0$ such that $h^{\prime}(x)$ is strictly increasing in $(0, a)$ and strictly decreasing in $(a, \infty)$.

Note that Lemma 3 implies that $h^{\prime}(x)$ cannot be constant in any interval, so the strictly increasing or decreasing function assumptions in the statement of Corollary 1 are without loss of generality. Although Corollary 1 is valid for functions having positive derivatives, a symmetric result can be proven for functions with negative derivatives [19]. Corollary 1 is a general statement also covering unbounded functions, although the functions we are particularly interested in are bounded.

\section{Remark 1}

Let $h$ be a function satisfying the assumptions of Corollary 1. Moreover, supposing that $h$ is bounded, then $h^{\prime}$ cannot be a strictly increasing function. Because if $h^{\prime}$ is a strictly increasing 

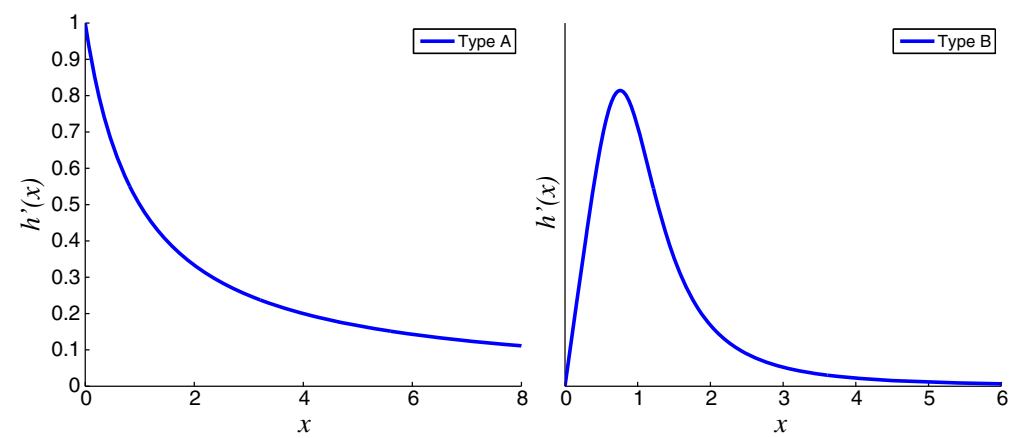

Figure 2. Typical $h^{\prime}(x)$ vs $x$ graphs for types A and B functions.

function, then $h$ cannot be bounded. Therefore, for a bounded function $h$ with a negative Schwarzian derivative, either $h^{\prime}$ is a strictly decreasing function in $[0, \infty]$ or there exists $a \geqslant 0$ such that $h^{\prime}$ is strictly increasing in $(0, a)$ and strictly decreasing in $(a, \infty)$.

Remark 1 leads us to the following definition:

\section{Definition 1}

For a bounded function $h$ with a negative Schwarzian derivative, we will say $h$ is of type A if $h^{\prime}$ is a strictly decreasing function, and of type B otherwise. The two types of such functions are generically illustrated in Figure 2.

Also note that whether the function $h$ is of type A or B, we always have

$$
\lim _{x \rightarrow \infty} h^{\prime}(x)=0
$$

Remark 2

Suppose the function $h$ is defined as follows:

$$
h(x)=g \circ g(x),
$$

where $g(x)$ is a function defined from $\mathbb{R}_{+}$to $X \subseteq \mathbb{R}_{+}$such that

$$
S g(x)<0 \quad \text { and } \quad g^{\prime}(x)<0 \quad \forall x \in(0, \infty) .
$$

Then, by the convolution property of the Schwarzian derivative, we have

$$
\operatorname{Sh}(x)<0 \quad \forall x \in(0, \infty) .
$$

Moreover, if $x_{0}$ is a fixed point of $h$, then one of the following holds:

1. $x_{0}$ is a fixed point of $g$.

2. $x_{0}<g\left(x_{0}\right)$, so $h\left(g\left(x_{0}\right)\right)=g\left(g\left(g\left(x_{0}\right)\right)\right)=g\left(x_{0}\right)$ and $h$ has another fixed point $>x_{0}$.

3. $g\left(x_{0}\right)<x_{0}$, so $h\left(g\left(x_{0}\right)\right)=g\left(g\left(g\left(x_{0}\right)\right)\right)=g\left(x_{0}\right)$ and $h$ has another fixed point $<x_{0}$.

Therefore, if $g$ has even number of fixed points, then $h$ has even number of fixed points; if $g$ has odd number of fixed points, then $h$ has odd number of fixed points. Also note that the conditions presented in Remark 2 are valid for Hill functions as well as for the tangent hyperbolic function.

\section{Proposition 1}

Let $g: \mathbb{R}_{+} \rightarrow \mathbb{R}_{+}$be a function such that $\forall x \in \mathbb{R}_{+}$, we have $S g(x)<0$ and $g^{\prime}(x)<0$. For each $x$, let $h(x)=g \circ g(x)$. Suppose $x_{0}$ is the unique fixed point of the function $g$. Then, we have the following:

1. If $\left|g^{\prime}\left(x_{0}\right)\right|<1$, then $h$ has the unique fixed point $x_{0}$.

2. If $h$ is of type $\mathrm{A}$, then $h$ has the unique fixed point $x_{0}$ satisfying $h^{\prime}\left(x_{0}\right)<1$. 


\section{If $h$ is of type $\mathrm{B}$ and}

(i) $h^{\prime}\left(x_{0}\right)<1$, then $h$ has the unique fixed point $x_{0}$.

(ii) $h^{\prime}\left(x_{0}\right)>1$, then $h$ has exactly three fixed points.

Proof

See the Appendix.

Let us assume that the condition $h^{\prime}\left(x_{0}\right)>1$ is satisfied, and let $y$ and $z$ denote the fixed points of $h$ different from $x_{0}$. From Remark 2, $x_{0}$ must be between $y$ and $z$, say

$$
y<x_{0}<z
$$

From the arguments used in the proof of Proposition 1, we can prove that

$$
f^{\prime}(y) \leqslant 1 \text { and } f^{\prime}(z) \leqslant 1 .
$$

\section{Remark 3}

In Proposition 1, we assumed that $h$ is in the form (21). Even if $h$ does not have this special form, the conclusion that $h$ has at most three fixed points will still be valid, but in this case, $h$ may have two fixed points.

\section{APPLICATIONS TO GENE REGULATORY NETWORKS}

In this section, we apply the results of Section 4 to the GRN described by the system of equations (3) under negative feedback; that is, $g$ defined in (5) satisfies the following:

$$
g^{\prime}(x)<0, \quad \forall x \in(0, \infty) .
$$

So, $g$ has a unique fixed point, and hence, the system (3) has a unique equilibrium point, which is denoted as $x_{e q}=\left(x_{1}, \ldots, x_{n}\right)$.

\section{Lemma 4}

For the system (3), $\mathbb{R}_{+}^{n}$ is a positively invariant set. Moreover, $x(t)$ remains bounded for any $x(0) \in \mathbb{R}_{+}^{n}$.

\section{Proof}

To prove positive invariance, we only need to check the direction of the vectors on the boundaries of the region $\mathbb{R}_{+}^{n}=\left\{\left(x_{1}, x_{2}, \ldots, x_{n}\right) \in \mathbb{R}^{n}: x_{i} \geqslant 0 \quad \forall i=1,2, \ldots, n\right\}$. The boundaries of the region $\mathbb{R}_{+}^{n}$ are just the planes $x_{i}=0$. If $x_{i}=0$ for some $i=1, \ldots, n$, then we have $\dot{x}_{i}=f\left(x_{i-1}\right) \geqslant 0$; so if the derivative is zero, $x_{i}$ stays on the boundary; otherwise, $x_{i}$ moves inside the region $\mathbb{R}_{+}^{n}$, which shows that the region $\mathbb{R}_{+}^{n}$ is an invariant set of the system (3). For the second part of the lemma, note that if $x_{i}(t)$ becomes greater than $\frac{1}{\lambda_{i}} \sup _{a \geq 0}\left|g_{i}(a)\right|$, then we have $\dot{x}_{i}(t)<0$, which means that $x_{i}(t)$ is decreasing. Hence, the solutions remain bounded for any positive initial condition.

Let $x_{e q}=\left(x_{1}, \ldots, x_{n}\right)$ be the unique equilibrium point of our system. Then, we have the following linearization of system (3) around its unique equilibrium point $x_{e q}$ :

$$
\dot{x}(t)=A_{0} x(t)+A_{1} x(t-\tau),
$$

where

$$
A_{0}=\left[\begin{array}{ccccc}
-\lambda_{1} & g_{1}^{\prime}\left(x_{2}\right) & 0 & \ldots & 0 \\
0 & -\lambda_{2} & g_{2}^{\prime}\left(x_{3}\right) & \ldots & 0 \\
\vdots & \ddots & \ddots & \ddots & \vdots \\
0 & 0 & \ldots & \ldots & -\lambda_{n}
\end{array}\right], \quad A_{1}=\left[\begin{array}{ccc}
0 & \ldots & 0 \\
\vdots & \ddots & \vdots \\
g_{n}^{\prime}\left(x_{1}\right) & 0 & \ldots
\end{array}\right]
$$


which gives us a characteristic equation of the following form:

$$
\chi(s)=\left(\prod_{i=1}^{n}\left(s+\lambda_{i}\right)\right)+k e^{-\tau s} \quad \text { where } \quad k=\left(\prod_{i=1}^{n-1} g_{i}^{\prime}\left(x_{i+1}\right)\right) g_{n}^{\prime}\left(x_{1}\right) .
$$

Because we have $\lambda_{i}>0$, the characteristic function $\chi(s)$ defined in (27) has all its roots in $\mathbb{C}_{-}$if and only if the transfer function

$$
G(s):=\left(1+\frac{k e^{-\tau s}}{\prod_{i=1}^{n}\left(s+\lambda_{i}\right)}\right)^{-1}
$$

is stable. Then, we have the following Lemma:

\section{Lemma 5}

Let $G(s)$ be as defined in (28), then $G(s)$ is stable independent of delay if

$$
\left|g^{\prime}\left(x_{1}\right)\right|<1
$$

Proof

By applying a small gain argument [20], we see that $G(s)$ is stable independent of delay if we have

$$
|k|<\left(\prod_{i=1}^{n} \lambda_{i}\right)
$$

Note that at the unique equilibrium $x_{e q}=\left(x_{1}, \ldots, x_{n}\right)$ of the system (3), we have

$$
g\left(x_{1}\right)=x_{1} ;
$$

that is, $x_{1}$ is the unique equilibrium point of the function $g(x)$. Then, observe that

$$
|k|=\left|\left(\prod_{i=1}^{n-1} g_{i}^{\prime}\left(x_{i+1}\right)\right) g_{n}^{\prime}\left(x_{1}\right)\right|=\left|g^{\prime}\left(x_{1}\right)\right|\left(\prod_{i=1}^{n} \lambda_{i}\right) .
$$

Hence, (29) and (31) imply (30); so, we obtain the desired result by the small gain theorem.

\section{Lemma 6}

Let $G(s)$ be defined as in (28); then $G(s)$ is unstable independent of delay if

$$
\left|g^{\prime}\left(x_{1}\right)\right|>1
$$

Proof

Because the system (3) is under negative feedback and $\left|g^{\prime}\left(x_{1}\right)\right|>1$, we have

$$
k<-\prod_{i=1}^{n} \lambda_{i}<0
$$

We can see that no matter which positive value the delay takes, there will be always a pole of $G(s)$ on the positive real axis. Therefore, system (3) will always be unstable for this case.

To continue our analysis, we need the following adoption of Theorem 1 in [21]:

Theorem 2

Consider the system (3) under assumptions 1 and 2 and suppose that $g$ defined in (5) is decreasing. Let $x_{e q}$ be the unique equilibrium point of the system (3). If the function $g \circ g$ has a unique positive fixed point, then for any nonnegative initial condition we have

$$
\lim _{t \rightarrow \infty} x(t)=x_{e q}
$$


If the function $g \circ g$ has more than one but finitely many positive fixed points and $l$ and $L$ are the lower and upper bounds of these fixed points, then for any solution $x(t)=\left(x_{1}(t), x_{2}(t), \ldots, x_{n}(t)\right)$ of the system (3) we have

$$
l<\lim _{t \rightarrow \infty} \underline{x_{i}(t)} \leqslant \lim _{t \rightarrow \infty} \overline{x_{i}(t)}<L \quad \forall i=1,2, \ldots, n .
$$

Theorem 2 leads to the following result:

\section{Proposition 2}

Consider system (3) under negative feedback and suppose that Assumptions 1 and 2 hold. Let $g$ be defined as in (5). Then, the system has a unique equilibrium point given by $x_{e q}=\left(x_{1}, \ldots, x_{n}\right)$, where $x_{1}$ is the unique fixed point of the function $g$. Moreover, if

$$
\left|g^{\prime}\left(x_{1}\right)\right|<1,
$$

then for any nonnegative initial condition the solution satisfies

$$
\lim _{t \rightarrow \infty} x(t)=x_{e q}
$$

Proof

By Lemma 2, it is easy to see that the system has a unique equilibrium point in the form $x_{e q}=\left(x_{1}, \ldots, x_{n}\right)$, where $x_{1}$ is the unique fixed point of the function $g$. Now, by Theorem 2, we obtain the desired result if we can show that the function

$$
f(u)=g(g(u))
$$

has a unique fixed point. Because the nonlinearity functions $g_{i}(x)$ have negative Schwarzian derivatives, the functions $g(x)$ and $f(x)$ have negative Schwarzian derivatives by Lemma 1. Hence, the function $f(x)$ is in the form of the function $h$ defined in Proposition 1. Hence, if we have

$$
\left|g^{\prime}\left(x_{1}\right)\right|<1,
$$

then by Proposition 1 we conclude that the function $f(u)$ has a unique fixed point that is at the same time the unique fixed point of the function $g(x)$. Because the function $f(u)$ has a unique fixed point, the desired result follows from Theorem 2 .

Note that the condition $\left|g^{\prime}\left(x_{1}\right)\right|<1$ also corresponds to the delay-independent stability of the linearized system around the unique equilibrium point. Therefore, Proposition 2 is consistent with the result of Lemma 5. Most of the nonlinearity functions considered in biological systems do have negative Schwarzian derivatives, including the Hill functions. Therefore, the results we have in Proposition 2 are useful not only for the analysis of GRNs but also for other biological processes, for example, hematopoiesis [22]. After the global stability condition given in Proposition 2, we will present another result regarding the oscillatory behavior of system (3).

We showed that the system (3) has a unique equilibrium point and all solutions with nonnegative initial conditions are bounded. Therefore, the results presented in Theorem 1 are valid for system (3) under negative feedback. Theorem 1 applied to our system leads us to the following result:

\section{Proposition 3}

Consider the system (3) under negative feedback with its unique equilibrium point $x_{e q}=$ $\left(x_{1}, \ldots, x_{n}\right)$. Suppose that each $g_{i}$ has negative Schwarzian derivative. Then, $g$ defined in (5) has the unique fixed point $x_{1}$. If we have

$$
\left|g^{\prime}\left(x_{1}\right)\right|>1
$$

then there exists periodic solutions of the system (3). Moreover, for this case, the function

$$
f(u)=g(g(u))=u
$$


has exactly three distinct fixed points. Let $y_{1}$ and $y_{2}$ be the two fixed points of the function $f$ other than $x_{1}$. Then, if $x(t)=\left(x_{1}(t), \ldots, x_{n}(t)\right)$ is the solution of the system with any positive initial condition, we have

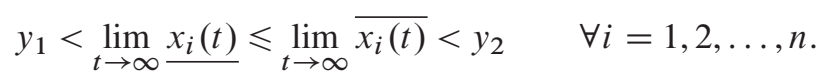

\section{Proof}

Theorem 1 implies that a solution $x(t)$ of the system (3) either converges to an equilibrium point or it is on a limit cycle. If we have $\left|g^{\prime}\left(x_{1}\right)\right|>1$, then

$$
k<\left(\prod_{i=1}^{n} \lambda_{i}\right)<0,
$$

which implies that the linearized system is unstable for all positive values of delay. So the equilibrium point is locally unstable independent of delay. Therefore, for some initial conditions around the unique equilibrium point $x_{e q}$, the corresponding solution of system (3) does not converge to $x_{e q}$. But for such initial conditions, if the solution does not converge to the unique equilibrium point $x_{e q}$, it can only be on a limit cycle by Theorem 1 . Therefore, system (3) has periodic solutions. It is easy to see that (35) implies (39).

Note that Proposition 3 gives not only a condition for the existence of periodic oscillations but also lower and upper bounds for possible periodic solutions of system (3). It can also be shown that [19]

$$
g^{\prime}\left(x_{1}\right) \neq-1
$$

so we either have $\left|g^{\prime}\left(x_{1}\right)\right|<1$ or $\left|g^{\prime}\left(x_{1}\right)\right|>1$, and hence, all possible cases have been studied.

\section{HOMOGENEOUS GENE REGULATORY NETWORKS WITH HILL FUNCTIONS}

In this section, we consider the homogenous GRN under negative feedback with Hill function type nonlinearities. In other words, we will analyze the following system:

$$
\left\{\begin{array}{l}
\dot{x}_{i}(t)=-x_{i}(t)+f\left(x_{i+1}(t)\right) \\
\dot{x}_{n}(t)=-x_{n}(t)+f\left(x_{1}(t-\tau)\right)
\end{array} \quad i=1,2, \ldots, n-1\right.
$$

where the function $f$ in (41) has the following form:

$$
f(x)=\frac{a}{b+x^{m}}, \quad a, b>0, \quad m \in\{1,2,3, \ldots\} .
$$

Notice that $f$ satisfies

$$
f^{\prime}(x)<0, \quad \forall x \in(0, \infty) .
$$

Also note that to have negative feedback, we should have odd number of interactions between genes. If $n$ is even, the system is under positive feedback, which is studied in [23]. That is, $n$ should be an odd number. Because $f(0)>0$ and $f(x)$ is decreasing, we conclude that $f$ has a unique fixed point, $x_{0}$, satisfying

$$
x_{0}=f\left(x_{0}\right)=\frac{a}{b+x_{0}^{m}} .
$$


Then, the unique equilibrium point of system (41) is $x_{e q}=\left(x_{0}, \ldots, x_{0}\right)$. Notice that we have

$$
f^{\prime}\left(x_{0}\right)=-\frac{m a x_{0}^{m-1}}{\left(b+x_{0}^{m}\right)^{2}}=-\frac{m x_{0}^{m+1}}{a} .
$$

From (43), we obtain the following equation:

$$
x_{0}^{m+1}=a-b x_{0} .
$$

Let us define a new function $h: \mathbb{R}_{+} \rightarrow \mathbb{R}_{+}$as

$$
h(x)=f^{n}(x) .
$$

Then, from Proposition 2, system (41) is stable if we have

$$
\left|h^{\prime}\left(x_{0}\right)\right|<1 \quad \Leftrightarrow \quad\left(\left|f^{\prime}\left(x_{0}\right)\right|\right)^{n}<1 .
$$

Combining (43) and (44), we obtain the following set of equations:

$$
\begin{aligned}
\left(\left|f^{\prime}\left(x_{0}\right)\right|\right)^{n}<1 & \Rightarrow\left|\left(f^{\prime}\left(x_{0}\right)\right)\right|<1 . \\
& \Rightarrow \frac{m x_{0}^{m+1}}{a}<1 \\
& \Rightarrow m x_{0}^{m+1}<a \\
& \Rightarrow m\left(a-b x_{0}\right)<a \\
& \Rightarrow \frac{(m-1) a}{m b}<x_{0} .
\end{aligned}
$$

Let $p: \mathbb{R}_{+} \rightarrow \mathbb{R}_{+}$be given by

$$
p(x)=x^{m+1}+b x-a .
$$

Clearly, $p\left(x_{0}\right)=0$ and we have

$$
p^{\prime}(x)=(m+1) x^{m}+b>0 \quad \forall x \in(0, \infty) \quad \text { and } \quad p(0)=-a<0 .
$$

Because $p\left(x_{0}\right)=0$ and $p$ is strictly increasing, we have

$$
p\left(\frac{(m-1) a}{m b}\right)<0 \Leftrightarrow \frac{(m-1) a}{m b}<x_{0} .
$$

We then have the following identities:

$$
\begin{aligned}
p\left(\frac{(m-1) a}{m b}\right) & =\left(\frac{m-1}{m}\right)^{m+1}\left(\frac{a}{b}\right)^{m+1}+\frac{m-1}{m} a-a \\
& =\left(\frac{m-1}{m}\right)^{m+1}\left(\frac{a}{b}\right)^{m+1}-\frac{a}{m} .
\end{aligned}
$$

Combining (50) and (51), we arrive at the following set of inequalities:

$$
\begin{aligned}
p\left(\frac{(m-1) a}{m b}\right) & <0 \\
& \Leftrightarrow\left(\frac{m-1}{m}\right)^{m+1}\left(\frac{a}{b}\right)^{m+1}<\frac{a}{m} \\
& \Leftrightarrow\left(\frac{a}{m}\right)^{m}<\left(\frac{b}{m-1}\right)^{m+1} .
\end{aligned}
$$

If the constants $a, b$ and $m$ satisfy the inequality (52), then from Proposition (2), the unique equilibrium point of system (41) is globally attractive. The arguments we had so far are valid 
for $m>1$ case. For $m=1$, the Hill functions do not have negative Schwarzian derivative. Now let $m=1$ and $a$ and $b$ arbitrary positive real numbers. Then, the system (41) has the unique equilibrium point $x_{e q}=\left(x_{0}, \ldots, x_{0}\right)$ satisfying

$$
x_{0}=\frac{a}{b+x_{0}} \Rightarrow x_{0}^{2}=a-b x_{0}
$$

From Proposition 2, the unique equilibrium point of (41) is globally attractive if

$$
\begin{aligned}
g^{\prime}\left(x_{0}\right) & =\frac{a}{\left(b+x_{0}\right)^{2}}=\frac{x_{0}^{2}}{a}<1 \\
& \Rightarrow a-b x_{0}<a \\
& \Rightarrow 0<x_{0} .
\end{aligned}
$$

Equation (53) shows that for $m=1$, the unique equilibrium point of (41) is globally attractive regardless of the values of the positive constants $a$ and $b$. Thus, the following result has been established.

\section{Proposition 4}

Consider system (41) and let $x_{e q}=\left(x_{0}, \ldots, x_{0}\right)$ be its equilibrium point.

(i) If $m=1$, then $x_{e q}$ is globally attractive for all positive constants $a, b$.

(ii) If $m=2,3, \ldots$ and $a, b, m$ satisfy

$$
\left(\frac{a}{m}\right)^{m}<\left(\frac{b}{m-1}\right)^{m+1}
$$

then $x_{e q}$ is globally attractive.

(iii) The system (41) has periodic solutions for cases other than (i) and (ii).

Proof

The proof comes from the fact that if (i) and (ii) are not satisfied, then the linearized system around its unique equilibrium point is unstable independent of delay. Hence, by using Theorem 1, we obtain the proposed conditions.

\section{EXAMPLES}

We now illustrate the theoretical results obtained in previous sections by a few illustrative examples.

Example 1

We consider system (3) with $n=3, \lambda_{1}=\lambda_{2}=\lambda_{3}=1$, and the nonlinearity functions are given by

$$
g_{1}(x)=\frac{1}{1+x} \quad g_{2}(x)=\frac{2}{2+x} \quad g_{3}(x)=\frac{1}{3+x} .
$$

The unique equilibrium point of the system can be found as $x_{e q}=(0.83,0.4,0.45)$, and at the unique fixed point of $g$, we have

$$
g^{\prime}(0.83)=0.0173<1 .
$$

Therefore, by Proposition 2, we expect that the solution converges to $x_{e q}$ independent of delay. Figure 3 shows the solution of the system with $x(0)=(1,0.9,0.8), \tau=0$. As expected, the solution converges to $x_{e q}$. Figure 4 is the solution of the same system with $x(0)=(0.4,2,0.6), \tau=4$. The solution again converges to $x_{e q}$. 


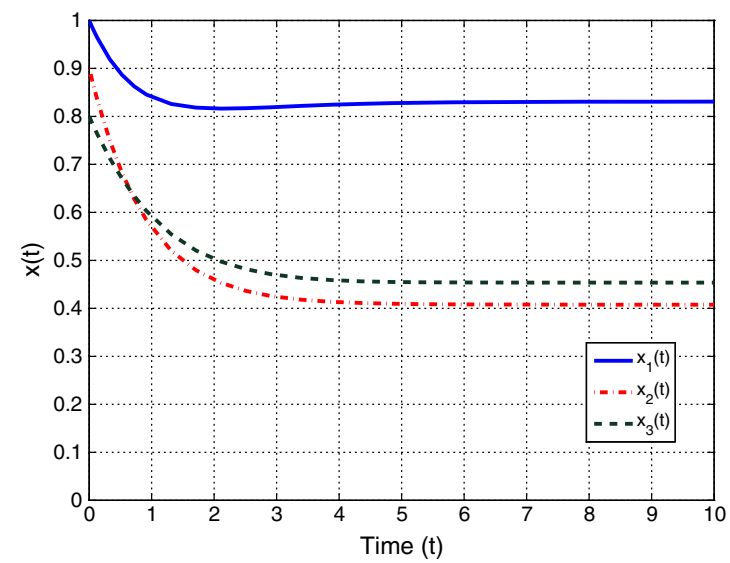

Figure 3. $x_{1}(t), x_{2}(t)$ and $x_{3}(t)$ vs $t$ graphs of the system with $x(0)=(1,0.9,0.8), \tau=0$.

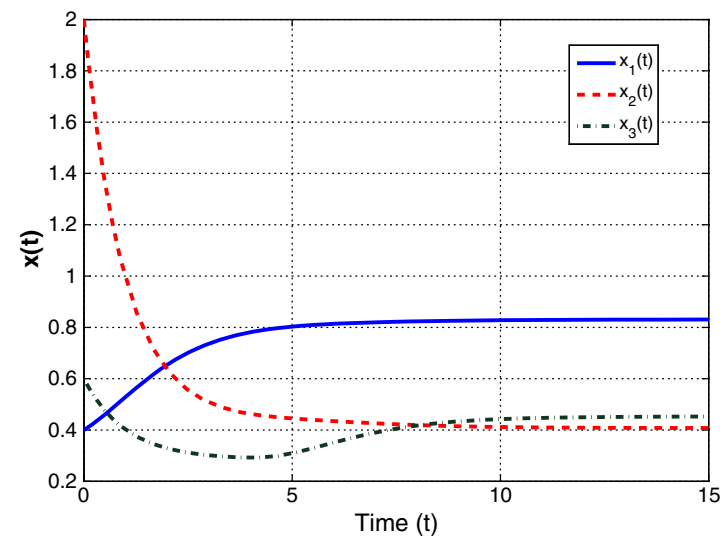

Figure 4. $x_{1}(t), x_{2}(t)$ and $x_{3}(t)$ vs $t$ graphs of the system with $x(0)=(0.4,2,0.6), \tau=4$.

\section{Example 2}

(Homogenous negative feedback case) In this example, we will try to illustrate the results of Proposition 4. Consider that the homogenous negative feedback case with $a=2, b=0.25, m=3, n=3$ and $f$ is given by

$$
f(x)=\frac{2}{0.25+x^{3}}
$$

Note that

$$
\left(\frac{a}{m}\right)^{m}=0.2963>\left(\frac{b}{m-1}\right)^{m+1}=0.00024
$$

The function

$$
h(x)=f \circ f(x)
$$

has three fixed points given by $y_{1}=0.0039, y_{2}=1.1442$ and $y_{3}=8$. From Proposition 4 and Theorem 2, we expect oscillatory solutions of the system and the following inequality to be satisfied:

$$
0.0039=y_{1}<\lim _{t \rightarrow \infty} \underline{x_{i}(t)} \leqslant \lim _{t \rightarrow \infty} \overline{x_{i}(t)}<y_{3}=8 \quad \forall i=1,2,3 .
$$

The simulation result corresponding to $x_{1}(t)$, with initial conditions $x_{1}(0)=1, x_{2}(0)=3$, $x_{3}(0)=4$ and $\tau=0$, are shown in Figure 5. The other two coordinates behave similarly. We have periodic solutions as expected, and the inequality in (58) is satisfied. 


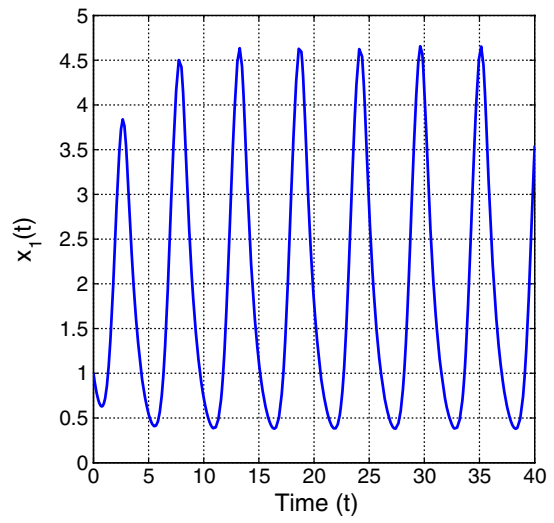

Figure 5. $x_{1}(t)$ versus $t$ graph of the system with $x(0)=(1,3,4), \tau=0$.

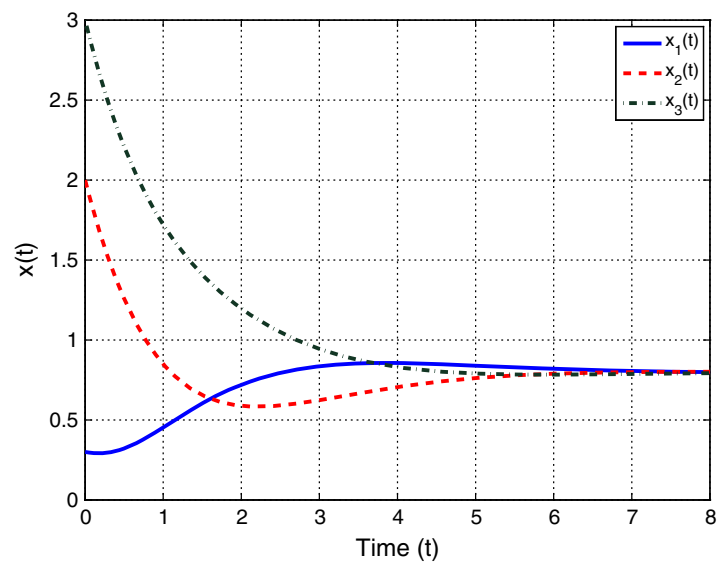

Figure 6. $x_{1}(t), x_{2}(t)$ and $x_{3}(t)$ versus $t$ graphs of the system with $x(0)=(0.3,2,3), \tau=0$.

Now consider the same problem with $a=2, b=2, m=3$ and $n=3$; then we have

$$
f(x)=\frac{2}{2+x^{3}} \quad \text { which implies }\left(\frac{a}{m}\right)^{m}=1<\left(\frac{b}{m-1}\right)^{m+1}=8 .
$$

In this case, the unique equilibrium point can be calculated as

$$
x_{e q}=(0.7709,0.7709,0.7709) .
$$

From Proposition 4, we expect the solution of the system to converge to $x_{e q}$ for any initial condition. The simulation result with three sets of initial conditions and time delays are shown in Figures 6-8. The simulation results confirms the following theory: $x(t)$ converges to $x_{e q}$ independent of delay in all the cases studied previously.

\section{CONCLUSIONS}

In this work, GRNs are considered; these are modeled as cyclic nonlinear dynamical systems with time delayed feedback. The negative feedback case is studied here; for the positive feedback case, see $[19,23]$. The nonlinearity functions are assumed to have negative Schwarzian derivatives. Such functions are then classified; and on the basis of this classification, results about the fixed points of such functions are obtained. Using these results on Schwarzian derivatives, we obtained global 


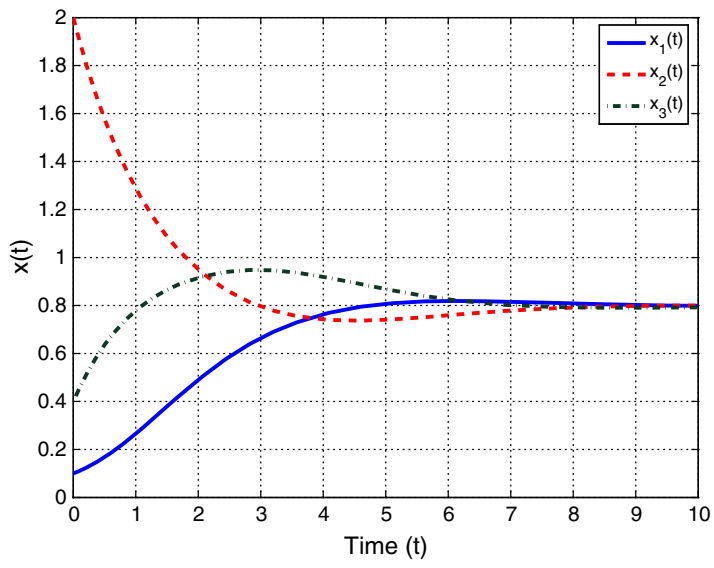

Figure 7. $x_{1}(t), x_{2}(t)$ and $x_{3}(t)$ versus $t$ graphs of the system with $x(0)=(0.1,2,0.4), \tau=1$.

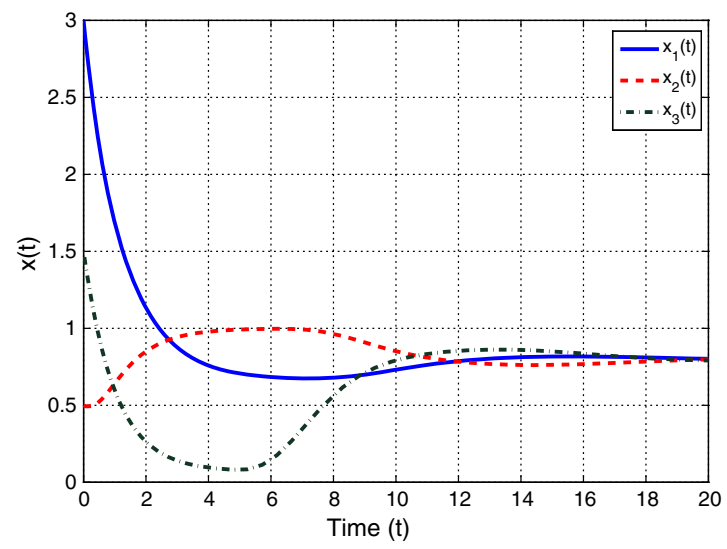

Figure 8. $x_{1}(t), x_{2}(t)$ and $x_{3}(t)$ versus $t$ graphs of the system with $x(0)=(3,0.5,1.5), \tau=5$.

stability conditions independent of delay for GRNs under negative feedback. The results are completed by an appropriate condition for instability independent of delay (leading to oscillatory behavior). The computation of a general expression for the period of oscillations is the subject of a future study. As a special case of the negative feedback, the homogenous GRNs are studied as well, where all nonlinearity functions are equal to a Hill function. For this case, a delay-independent global stability result is obtained depending only on the parameters of the Hill function. A similar result is given for the existence of oscillatory solutions.

\section{APPENDIX: PROOF OF PROPOSITION 1}

First, note that because $g$ is a strictly decreasing function, we have

$$
g(0)>g(x)>0 \quad \forall x>0,
$$

so $g(x)$ is a bounded function, which implies that the function $h$ is bounded. Because $g$ is a decreasing function, it has a unique fixed point $x_{0}$. Observe that

$$
h^{\prime}(x)=g^{\prime}(g(x)) g^{\prime}(x) .
$$

Because $g^{\prime}(x)<0 \quad \forall x \in(0, \infty)$, we have

$$
h^{\prime}(x)>0 \quad \forall x \in(0, \infty) .
$$


At the unique fixed point $x_{0}$ of $g$, we have the following equality:

$$
\begin{aligned}
& \qquad h^{\prime}\left(x_{0}\right)=g^{\prime}\left(g\left(x_{0}\right)\right) g^{\prime}\left(x_{0}\right)=\left(g^{\prime}\left(x_{0}\right)\right)^{2} . \\
& \text { Therefore, we have }\left|g^{\prime}\left(x_{0}\right)\right|<1 \quad \Leftrightarrow \quad h^{\prime}\left(x_{0}\right)<1, \\
& \text { and similarly, }\left|g^{\prime}\left(x_{0}\right)\right|>1 \quad \Leftrightarrow \quad h^{\prime}\left(x_{0}\right)>1 .
\end{aligned}
$$

We have shown that the function $h$ is either of type A or type B. Therefore, if we prove the second and third parts of the Proposition, then the first part follows straightforwardly. For the first part of the Proposition, assume that the function $h$ is of type A and then $h^{\prime}$ is strictly decreasing in $\mathbb{R}_{+}$. Notice that because $h$ is bounded, we have

$$
\lim _{x \rightarrow \infty}\left(h^{\prime}(x)\right)=0 .
$$

If $h^{\prime}\left(x_{0}\right) \geqslant 1$, then because $h^{\prime}$ is a decreasing function, we have

$$
h^{\prime}(x)>1 \quad \forall x \in\left[0, x_{0}\right] .
$$

From mean value theorem for some $t \in\left[0, x_{0}\right]$, we have the following:

$$
h^{\prime}(t)=\frac{h\left(x_{0}\right)-h(0)}{x_{0}} \leqslant \frac{x_{0}-h(0)}{x_{0}} \leqslant 1 .
$$

But, on the other hand, we have

$$
h^{\prime}(x)>1, \quad \forall x \in\left[0, x_{0}\right],
$$

so we arrived to a contradiction. Therefore, we have $h^{\prime}\left(x_{0}\right)<1$. Now, suppose there exists another fixed point of the function $h$. We know from Remark 2 this implies that

$$
\exists y \geqslant x_{0} \quad \text { such that } \quad h(y)=y .
$$

But mean value theorem implies that there exists $t \in\left[x_{0}, y\right]$ such that

$$
h^{\prime}(t)=\frac{h(y)-h\left(x_{0}\right)}{y-x_{0}}=1 .
$$

Because $h^{\prime}(x)$ is a strictly decreasing function, we have

$$
h^{\prime}(x)<1, \quad \forall x \geqslant x_{0} .
$$

Therefore, $h$ has the unique fixed point $x_{0}$. For the third part of the Proposition, we assume that $h$ is of type B. We define a new function in the following way:

$$
f(x)=x-h(x) .
$$

Then, clearly we have

$$
f(0)<0 \text { and } f^{\prime}(x)=1-h^{\prime}(x) .
$$

Note that the zero crossings of the function $f$ and the fixed points of the function $h$ are the same. Suppose that

$$
h^{\prime}\left(x_{0}\right)<1 .
$$

Also assume that the function $h$ has a fixed point $y$, which is different from $x_{0}$. From Remark 2, we can safely assume that

$$
y<x_{0} .
$$


Again from Remark 2, we have another fixed point of $h$, which is denoted by $z$ and is greater than $x_{0}$. For type B functions, we have either

$$
\begin{array}{ll}
h^{\prime}(x)<h^{\prime}\left(x_{0}\right)<1, & \forall x \in\left[0, x_{0}\right] \text { or } \\
h^{\prime}(x)<h^{\prime}\left(x_{0}\right)<1, & \forall x \in\left[x_{0}, \infty\right] .
\end{array}
$$

If the condition (67) is satisfied, then we have $f(0)<0$ and

$$
f^{\prime}(x)>0 \quad x \in\left[0, x_{0}\right] .
$$

Then, it is clear that $f(y)<0$; so, in other words, we have

$$
f(y) \neq 0
$$

which is a contradiction. For the case in (68) using a similar argument, we can show that $f(z) \neq 0$. Hence, if (65) is satisfied, then $h$ has the unique fixed point $x_{0}$. Now, let us assume that

$$
h^{\prime}\left(x_{0}\right)>1 .
$$

But for a type B function $h$, we can have at most two different values $t_{1}$ and $t_{2}$ such that

$$
h^{\prime}\left(t_{i}\right)=1 \quad \text { for } i=1,2 .
$$

Hence, $f$ can have at most three zero crossings, which implies that the function $h(x)$ has at most three fixed points. From (71), we can deduce the following:

$$
\exists x_{1}>x_{0} \text { such that } f\left(x_{1}\right)<0,
$$

but because the function $h$ is bounded, we have

$$
\lim _{t \rightarrow \infty}(f(x))=\infty .
$$

Therefore, $f$ has a zero crossing greater than $x_{0}$; thus, $h$ has a fixed point greater than $x_{0}$. But we know that the function $h$ has at most three fixed point. Thus, from Remark 2, we can conclude that $h$ has exactly three fixed points.

\section{ACKNOWLEDGEMENTS}

The authors wish to thank the Associate Editor and anonymous reviewers for their useful comments that helped us improve the overall quality of our paper. This work is supported in part by the French-Turkish PIA Bosphorus (TUBITAK Grant No. 109E127 and EGIDE Project No. 22974WJ) and by DPT-HAMIT project.

\section{REFERENCES}

1. Schena M. Genome analysis with gene expression microarrays. Bioessays 1996; 18:427-431.

2. Davidson E, Levin M. Gene regulatory networks. Proceedings of the National Academy of Sciences 2005; 102(14):4936-4942.

3. Jones PA, Baylin SB. The epigenomics of cancer. Cell 2007; 12(8):683-692.

4. Jong HD. Modeling and simulation of genetic regulatory systems: a literature review. Journal of Computational Biology 2002; 9:67-103.

5. Chen L, Aihara K. Stability of genetic regulatory networks with time delay. IEEE Transactions on Circuits and Systems 2002; 49(5):602-608.

6. Goldbeter A. Biochemical Oscillations and Cellular Rythms. The Molecular Basis of Periodic and Chaotic Behavior. Cambridge Univ. Press.: Cambridge, UK, 1996.

7. Roussel MA. The use of delay differential equations in chemical kinetics. Journal of Physical Chemistry 1996; 100:8323-8330.

8. Townley S, Ilchmann A, Weiss MG, Mcclements W, Ruiz DH, Owens D, Praetzel-Wolters D. Existence and learning of oscillations in recurrent neurela networks. IEEE Transactions on Neural Networks 2000; 11:205-214.

9. Sontag ED. Asymptotic amplitudes and Cauchy gains: a small-gain principle and an application to inhibitory biological feedback. Systems Control Let 2002; 47:167-179.

10. Chu D, Zabet NR, Mitavskiy B. Models of transcription factor binding: sensitivity of activation functions to model assumptions. Journal of Theoretical Biology 2009; 257:419-429. 


\section{ANALYSIS OF GENE REGULATORY NETWORKS UNDER NEGATIVE FEEDBACK}

11. Morărescu C-I, Niculescu S-I. Some remarks on the delay effects on the stability of biochemical networks. 16th Mediterranean Conference on Control and Automation, Ajaccio, France, 2008; 801-805.

12. Gebert J, Radde N, Weber GW. Modeling gene regulatory networks with piecewise linear differential equations. European Journal of Operational Research 2007; 181:1148-1165.

13. Müller S, Hofbauer J, Endler L, Flamm C, Widder S, Schuster P. A generalized model of the repressilator. Journal of Mathematical Biology 2006; 53:905-937.

14. Enciso GA. On the asymptotic behaviour of a cylic biochemical system with delay. Proceedings of the 45th IEEE Conference on Decision and Control, San Diego, CA, 2006; 2388-2393.

15. Angeli D, Sontag ED. Multistability in monotone input/output systems. Systems \& Control Letters 2004; 51:185-202.

16. Liz E, Pinto M, Robledo G, Trofimchuck S, Tkachenko V. Wright type delay differential equations with negative Schwarzian. Discrete and Continuous Dynamical Systems 2003; 9(2):309-321.

17. Sedaghat H. Nonlinear Difference Equations. Kluwer Academic Publishers: Norwell, MA, 2003.

18. Parret JM, Sell GR. The Poincaré-Bendixson theorem for monotone cyclic feedback systems with delay. Journal of Differential equations 1996; 125:441-489.

19. Ahsen ME. Analysis of two types of cyclic biological system models with time delays. MS Thesis, Graduate School of Engineering and Sciences, Bilkent University, Ankara, Turkey, July 2011.

20. Özbay H. Introduction to Feedback Control Theory. CRC Press: Boca Raton, FL, 2000.

21. Allwright DJ. A global stability criterion for simple control loops. Journal of Mathematical Biology 1977; 4(4):363-373

22. Mackey MC. Unified hypothesis of the origin of aplastic anaemia and periodic hematopoiesis. Blood 1978; 51:941-956.

23. Ahsen ME, Özbay H, Niculescu SI. Stability analysis of a dynamical model representing gene regulatory networks. 10th IFAC Workshop on Time Delay Systems, Boston, USA, June 2012; 191-196. 